\title{
Time to exhaustion at anaerobic threshold in swimming rats: metabolic investigation
}

\author{
Beck WR ${ }^{1,2}$, De Araujo GG ${ }^{3}$, Scariot $\mathrm{PPM}^{1}$, dos Reis IGM ${ }^{1,2}$, Gobatto $\mathrm{CA}^{1,2}$ \\ Laboratório de Fisiologia Aplicada ao Esporte, Faculdade de Ciencias Aplicadas, Universidade Estadual de Campinas, \\ Limeira, Sao Paulo, Brasil. cgobatto@uol.com.br
}

\begin{abstract}
The purpose of this study was to determine the time to exhaustion (tlim) for swimming exercise at anaerobic threshold (AT) intensity in rats and to analyze metabolic consequences on serum and tissues levels. Eighteen rats were divided in control (CG) and exercised (EG) groups, being the former submitted to tlim. We analyzed the glycogen content of liver and ten skeletal muscles, as well as serum parameters. Parametric statistic was used with significance level at $p<0.05$. The tlim, which was correspondent to $114.37 \pm 36.23$ min, promoted significant decrease in blood glucose $(42.99 \% ; p<0.01)$ and an increase in free fatty acids (167.12 \%; $p<0.01)$ when EG was compared to CG. We did not find differences in albumin, total protein uric acid and creatinine between groups. The proposed exercise at individualized AT intensity promoted severe glycogen depletion for all tissues (mean of $78.05 \%$ for all muscles and $89 \%$ for liver). With substantial control of exercise intensity, our study establishes a useful rodent model that can be further explored, contributing to the advancement on knowledge and better understanding of exhaustion mechanisms (Tab. 1, Fig. 2, Ref. 35). Text in PDF www.elis.sk.

Key words: aerobic capacity, anaerobic threshold, glycogen and glycolytic metabolism.
\end{abstract}

Physiological alterations generated by different effort have been the focus of exercise physiologists since many years. Moderate and heavy exercise produces physiological alterations (1), which are easily sustained by the organism. In this case, beyond $\beta$-oxidation, glycolytic energy is yielded through glucose to pyruvate reduction and then following the oxidative pathway in form of acetyl coenzyme A, producing proper amount of mitochondrial ATP. As exercise intensity increases, oxidative pathway is no longer able to yield all the energy demand, recruiting gradually the anaerobic pathway. Thus, lactate dehydrogenase forms lactic acid from pyruvate in skeletal muscle fibre cytoplasm when glycolytic rate increases (2), also increasing blood lactate, hydrogen ions and carbonic gas concentrations (1). The disproportional increase of blood lactate concentration in relation to effort intensity during an incremental exercise determines the transition between aerobic and anaerobic metabolisms, called anaerobic threshold (AT) (3).

${ }^{1}$ Laboratório de Fisiologia Aplicada ao Esporte, Faculdade de Ciencias Aplicadas, Universidade Estadual de Campinas, Limeira, Sao Paulo, Brasil, ${ }^{2}$ Faculdade de Educacao Fisica, Universidade Estadual de Campinas, Sao Paulo, Brasil, and ${ }^{3}$ Universidade Federal de Alagoas, Maceio, Alagoas, Brasil

Address for correspondence: C.A. Gobatto, Dr, Laboratório de Fisiologia Aplicada ao Esporte, Faculdade de Ciencias Aplicadas, Universidade Estadual de Campinas. Endereco, Rua Pedro Zaccaria, 1.300, Jardim Santa Luíza - CEP: 13484-350 - Limeira - Sao Paulo.

Phone: +19.3701.6653, Fax: +19.3701.6680

Acknowledgements: This study was supported by the Sao Paulo State Research Support Foundation (FAPESP) Proc. 2010/13377-7 and 2011/13226-1.
AT is generally determined by protocols with approximately 30 or 40 minutes, but it is know that at this intensity the subject can sustain exercise for more time until exhaustion (4). As a result, the $\mathrm{AT}$ is an indirect procedure to determine the aerobic endurance and not always leads to exhaustion, while the time to exhaustion at this intensity (tlim) is adopted as a direct procedure (5). The motives of exhaustion at AT has been investigated since the 1920s, and owing the difficulty caused by multi factorial characteristic, are still actively researched today (6). Some authors have associated the inability to continue exercise at aerobic required intensity to a lack of energetic substrates, mainly glycogen depletion $(7,8)$. Beneke, et al. (9) postulated that glycogen is the primary energy substrate during maximal aerobic capacity, and its depletion is likely the principal factor preventing continued aerobic exercise (10).

In order to test this glycogen paradigm and characterize tlim consequences, we employed an animal model of swimming exercise (11), an excellent way to study physiological, cardiovascular, pulmonary and metabolic mechanisms (7). These animals show high similarities regarding blood lactate-intensity relationship comparing to humans beings, eliciting accurate quantification of exercise intensity (12-15). Ahead of this, the aim of this study was to determine tlim at AT and analyze such consequences through serum metabolic variables, glycogen content of liver and mapping ten skeletal hindlimb muscles.

\section{Materials and methods}

\section{Animals}

Eighteen male Wistar rats (rattus novergicus) were housed in polypropylene cages (four or five rats per cage) and kept at 
$617-621$

$22 \pm 1{ }^{\circ} \mathrm{C}$ in $45-55 \%$ relative humidity with noise always below 80 decibels. Was established a photoperiod of 12 hours light (6:00-18:00 h) / 12 hours dark and 60 lux was the maximal light intensity inside cage (Phillips ${ }^{\circledR}$ soft white light lamp, 100W, 2700 K, 565-590 nm). Standard food (23.5\% protein, $6.5 \%$ fat, $70 \%$ carbohydrate, Purina 5008, St. Louis, MO) and water were available ad libitum. The experiment was conducted according to the Brazilian College for Animal Experimentation and was approved by Ethics Committee on the Use of Animals of Bioscience Institute of Sao Paulo State University under the protocol number 018/2010.

\section{Experimental procedures and swimming routines}

The animals were housed in the environment described above with 45 days old. Two weeks before turning 90 days old, they began a general adaptation period for the experimental protocol, including exposure to aquatic environment, swimming in deep water ( $15-50-120 \mathrm{~cm}$ progressively) and handling for all procedures. All swimming routines occurred in an individual tank with $30 \mathrm{~cm}$ diameter and $120 \mathrm{~cm}$ deep, containing clean water at $31 \pm 1{ }^{\circ} \mathrm{C}$. We divided equally and randomly the animals into control (CG) and exercised (EG) groups. All underwent the lactate minimum test (LMT) at 90 days old as previously described (12), being determined the lactate minimum intensity (LMi, \% body weight), which is corresponding to AT and maximal lactate steady state in swimming rats (13). Forty-eight hours after, rats were euthanized in carbon-dioxide chamber at rest (CG) or immediately after tlim (EG).

All procedures were initiated at 20:00 h, when the body temperature and natural activity of the rat is high $(14,16)$ and were carried out under red light (Phillips, Infrared-PAR38-E27-150W, wavelength $>600 \mathrm{~nm}, \sim 15$ lux) considering that albino Wistar rats are very photosensitive $(16,17)$. This time of day was also selected by considering the optimum circadian period in order to minimize animal stress (18). The swimming exercise performed by EG was one bout at lactate minimum intensity, maintained until it can no longer be sustained (tlim). The tlim was verified when the rat remains submerged for at least 10 seconds despite vigorous attempts to emerge (11). These routines were conducted by the same two researchers and the experimental routines sequence is illustrated in Figure 1.

\section{Collection of biological material}

Blood samples $(25 \mu \mathrm{L})$ were collected in heparinized capillary tubes from the distal tail during LMT, before (pre [lac]) and immediately after (post [lac]) the tlim. These samples were immediately transferred to plastic tubes $(1.5 \mathrm{~mL})$ containing 400 $\mu \mathrm{L}$ of trichloroacetic acid (4\%) for the determination of lactate concentration, which was measured at $340(\mathrm{~nm})$ against the calibration curve (19).

Immediately after euthanasia, we performed a thoracotomy followed by blood sample collection by cardiac puncture. The obtained samples were centrifuged and each serum sample was assessed for albumin (ALB), creatinine (CREAT), total protein (TP), uric acid (UA), free fat acid (FFA) and glucose (GLUC), using commercial kits. We also extracted 10 skeletal muscles: gluteus

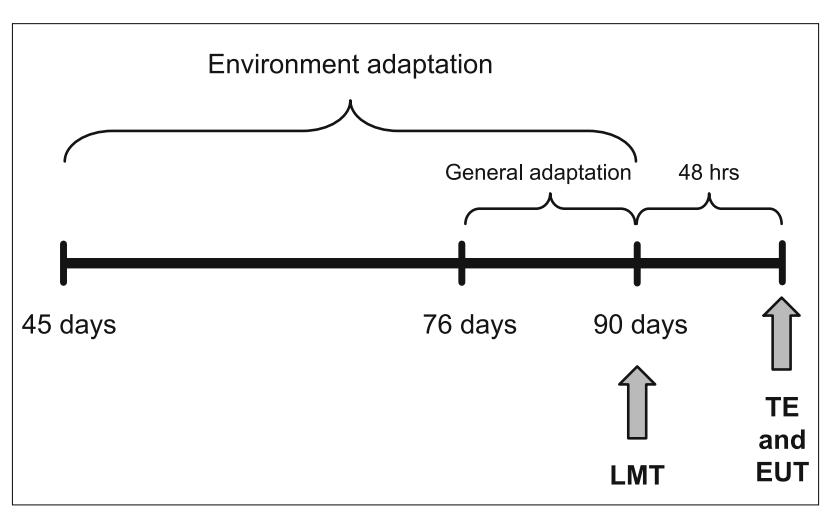

Fig . 1. Schematic summary of study design. Forty-five-day old animals were housed at bioterium. At 76 days old, the rats were submitted to general adaptation for two weeks. At 90 days old, the lactate minimum test (LMT) was applied. After an interval of $48 \mathrm{~h}$, the animals were euthanized (EUT) immediately after time to exhaustion (tlim) at lactate minimum intensity (exercised group) or at rest (control group).

maximus (GLU), biceps femoris (BF), soleus (SO), semimembranosus (SM), vastus lateralis (VL), semitendinous (ST), vastus intermedius (VI), vastus medialis (VM), rectus femoris (RF) and gastrocnemius (GTN). The tissue samples were prepared and analyzed by spectrophotometry at $490 \mathrm{~nm}$ by the colorimetric method, described elsewhere (20) to determine glycogen concentration. Liver glycogen was analyzed by the same method.

\section{Statistical procedures}

Values are the means \pm standard deviation. Normal Gaussian distribution of all data samples was verified with the Kolmogorov-Smirnov test before parametric statistical procedures were employed. We used independent t-tests to evaluate group differences in serum parameters and liver glycogen. ANOVA one way for repeated measures was employed to analyze the differences between muscles inside each group and ANOVA one way for independent samples was used for groups differences (CG vs EG) in each skeletal muscle. When necessary $(p<0.05)$, NewmannKeuls post hoc tests was used to specify differences. We used the Pearson product-moment correlation test to assess relationships between variables for the same group. The significance level was set at $\mathrm{p}<0.05$ for all.

\section{Results}

We found no significant differences between groups for LMi ( $p>0.05$ ), which was correspondent to $5.09 \pm 0.46 \%$ body weight ( $\mathrm{n}=18, \mathrm{CG}$ and EG). tlim was $114.37 \pm 36.23 \mathrm{~min}$, with pre[lac] and post[lac] corresponding to $1.55 \pm 0.33$ and $5.80 \pm 1.96 \mathrm{mM}$, respectively.

We observed a significant decrease in blood glucose (42.99\%) and an increase in FFA (167.12\%) in EG when compared to CG, being found a significant and negative correlation between these variables for EG $(r=-0.82 ; \mathrm{p}=0.013)$. Regarding albumin, total protein, creatinine and uric acid levels, no significant differences were found between groups (Tab. 1). 
Tab. 1. Albumin (ALB), total protein (TP), creatinine (CREAT), uric acid (UA), free fatty acid (FFA) and glucose (GLUC) serum concentrations for the control (CG) and exercised group (EG) expressed in the mean \pm standard deviation and independent $t$-test results (P).

\begin{tabular}{|c|c|c|c|c|c|c|}
\hline & $\operatorname{ALB}(g / d L)$ & $\mathrm{TP}(\mathrm{g} / \mathrm{dL})$ & CREAT (mg/dL) & $\mathrm{UA}(\mathrm{mg} / \mathrm{dL})$ & $\mathrm{FFA}(\mu \mathrm{E} / \mathrm{L})$ & GLUC (mg/dL) \\
\hline$\overline{\mathrm{CG}}$ & $3.15 \pm 0.68$ & $6.74 \pm 0.50$ & $1.22 \pm 0.37$ & $5.20 \pm 1.42$ & $256.72 \pm 108.95$ & $137.53 \pm 16.14$ \\
\hline $\mathrm{EG}$ & $3.48 \pm 0.33$ & $6.44 \pm 0.37$ & $1.29 \pm 0.36$ & $4.92 \pm 1.82$ & $685.75 \pm 159.39 *$ & $78.40 \pm 32.91 *$ \\
\hline $\mathrm{p}$ & 0.20 & 0.17 & 0.69 & 0.73 & $<0.01$ & $<0.01$ \\
\hline
\end{tabular}

$* \mathrm{p}<0.05$ in relation to $\mathrm{CG}$

Muscle glycogen concentration was significantly affected by exercise, and ANOVA tests reveal that the exercised group had a lower concentration than the control group when considered all skeletal muscles $\left(\mathrm{F}_{1,157}=221.67, \mathrm{p}<0.01\right)$. Were found significant differences on glycogen content among many muscles in the CG, however, these were not found for EG, where any of the ten muscles assessed showed statistical differences $(p>0.05$ for all post hoc crosses). Significant differences were found in some muscle glycogen concentrations when analyzed among groups (independent samples) and these values were used to calculate the percentage depletion of glycogen $(\% \mathrm{DG})$. These results for each muscle are shown in Figure 2, being that the mean depletion in all muscles was $78.05 \%$. The liver glycogen content were $5046.97 \pm 3801.24$ and $554.96 \pm 351.91 \mu \mathrm{g} / 100 \mathrm{mg}$ for CG and EG, respectively, being found statistical difference $(\mathrm{p}<0.01)$ and $89 \% \mathrm{DG}$.

\section{Discussion}

The main findings of the present study were the determination of the individual tlim in swimming rats, the significant muscle and liver glycogen depletion and the interesting responses on serum biochemistry of the animals owing this exercise.

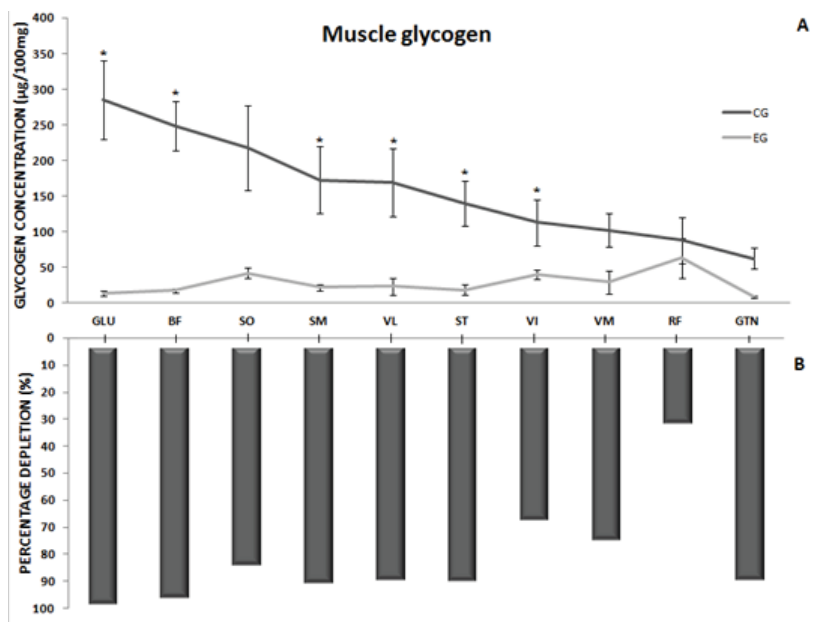

Fig. 2. A) Glycogen content $(\mu \mathrm{g} / 100 \mathrm{mg})$ of gluteus maximus (GLU; $p$ $<0.01$ ), biceps femoris (BF; $p<0.01$ ), soleus ( $\mathrm{SO} ; \mathrm{p}<0.01$ ), semimembranosus ( $\mathrm{SM} ; \mathrm{p}<0.01$ ), vastus lateralis ( $\mathrm{VL} ; \mathrm{p}<0.01)$, semitendinous (ST; $p<0.01)$, vastus intermedius (VI; $p<0.01)$, vastus medialis (VM; $p=0.086)$, rectus femoris $(\mathrm{RF} ; \mathrm{p}=\mathbf{0 . 2 4 5})$ and gastrocnemius $(\mathrm{GTN} ; \mathrm{p}=$ 0.705) of control group (CG) and experimental group (EG); B) Percentage of glycogen depletion (\%DG), considering initial glycogen content of CG and final glycogen content of EG. * $p<0.05$ in relation to EG.

\section{Performance}

The ability of the subject to resist fatigue is responsible for exercise duration with a constant intensity. In humans, tlim varies from 30.0 to $65.2 \mathrm{~min}(4,21)$. For instance, we did not aim to direct comparisons about tlim of humans and rats, however, our results suggest that rats can sustain exercise at AT longer before task failure. Although a lack of other data on rats' tlim prevents any comparison, we should note that our protocol was implemented at an ideal point in the rat circadian rhythm (dark period), under objective and individualized intensity control, environmental conditions, beyond the adaptation to handling and testing. It is possible that these conditions caused less stress in the animals (18), eliciting better results. The relatively high tlim in rats may also be due to the fact that the rats really must swim until exhaustion, whereas humans can stop because any discommodity in laboratory tests. Despite studies using LMT parameters for rat training prescriptions are designed to enhance performance or for drugfree diseases treatment $(13,22)$, the literature does not contain data regarding tlim. These results of our study may be useful for volume training prescription for swimming rats, where $\sim 50 \%$ of tlim represents approximately 55-60 minutes.

\section{Serum parameters}

Serum total protein and albumin levels after tlim suggested no changes in nutritional status or hydration (23), which allowed us to reject these parameters as factors that limit continued exercise. Creatinine scores were also not significantly affected by exercise either, so the tlim did not induce acute kidney problems or significantly alter creatinine metabolism.

Another parameter analyzed was uric acid (UA), which arose due to an inadequate rephosphorylation rate of ADP (adenosine diphosphate) to ATP (adenosine triphosphate) resulting in an increased concentration of AMP (adenosine monofhosphate) (24), which is likely converted to IMP (inosine monophosphate) and finally to UA (25). Nevertheless, UA values in our study did not suggest a significant difference between groups, leading to hypothesized that the cycle was not significantly complete. Serum parameters showed that protein metabolism remained unchanged after tlim.

Unlike protein parameters, glucose and lipids levels were significantly affected by exercise. In the EG, there was a glucose decrease of $42.99 \%$ and an FFA increase of $167.12 \%$ compared to the CG. The results corroborate with the literature; during aerobic exercise, the body has a tendency to "save" glucose (26) as a mechanism to slow glycogen oxidation, which increases FFA levels (27). However, glycogen is the principal substrate for exercise at aerobic intensity (9), being the blood glucose recruited, 
$617-621$

which resulted in considerable differences one or two hours after continuous effort (28). Moreover, Curi, et al (27) postulated that even when provided with FFA, muscle was unable to oxidize acetyl-CoA generated during $\beta$-oxidation, and as a consequence, ATP production was reduced and suggested that the exhaustion of the Krebs cycle was a determining factor of exercise exhaustion.

\section{Glycogen depletion}

Lactate concentration is not associated as responsible for exhaustion at AT intensity and Baron et al (21) postulated that fatigue at maximal lactate steady (AT parameter) state did not occur physiologically. Some authors postulated that failures in the muscle contraction mechanism were established before glycogen content was depleted completely (29), whereas others ascribed these failures to glycogen depletion $(7,8)$.

Our finding showed that glycogen concentrations were different between several muscles before exercise $(p<0.05)$, likely due to their individual characteristics. Nevertheless, after performance (tlim) glycogen content was equal when comparing all muscles $(p>0.05)$. These results suggested a uniform glycogen depletion, regardless of muscle fibre type, reinforcing the usefulness of the present model. This dramatic glycogen depletion, principally liver, could be responsible for the observed blood glucose, which interfered with central nervous system function and led to exhaustion via mechanisms that are not entirely understood (30).

Grounded in the nomenclature described by Peter et al (31), rat hindlimb muscle fibres are $76 \%$ of glycolitic (IIb), $19 \%$ oxidative glycolitic (IIa) and $5 \%$ oxidative (I) (32) and the progressive order of activation seems to be I, IIa and IIb (33). Neural drive it is not constant during exercise (34), and the recruitment of new fibre begins when others are fatigued (35). Nevertheless, this discussion was weakened by our observation that all muscles showed a high glycogen depletion independent of fibre type. The mean of EG glycogen concentration, computing all muscles, was $20 \mu \mathrm{g} / 100 \mathrm{mg}$, representing about $78.05 \%$ depletion and seems to be expressive. The less depleted was rectus femoris, but this muscle consists of $\sim 99 \%$ glycolytic fibres type (32) and retained only $62.70 \mu \mathrm{g} / 100 \mathrm{mg}$ of glycogen content at the end of exercise, showing an increase in neural drive (to achieve this fibre) over time at the same intensity. Gastrocnemious and vastus medialis also obtained $p>0.05$ when comparing CG and EG, likely because of their low glycogen concentration at rest (CG), generating a lack of significant statistics, however, depleting $71.59 \%$ and 86.36 $\%$, respectively. These three muscles seem to be less important to swimming exercise in front of others.

In summary, the time to exhaustion at anaerobic threshold intensity (tlim) in swimming rats was $114.37 \pm 36.23 \mathrm{~min}$. Despite the fact that we did not find modulation on serum protein parameters, the glycidic and lipidic serum variables showed significant variations. On the other hand, we found that all determined skeletal muscle glycogen were severely depleted after exercise (average value: $2 \mu \mathrm{g} / 100 \mathrm{mg}$ ) as well as liver glycogen content (89\%), showing that it had to be considered at least as one of the causes of exercise exhaustion at maximal aerobic intensity in swimming rats. With substantial control of exercise intensity, our study estab- lishes a useful rodent model that can be further explored, contributing to the advancement on knowledge and better understanding of exhaustion mechanisms during aerobic exercise.

\section{References}

1. Wasserman K, Van Kessel AL, Burton GG. Interaction of physiological mechanisms during exercise. J Appl Physiol 1967; 22 (1): 71-85.

2. Gladden LB. Lactate transport and exchange during exercise. Handbook of Physiology (ed) Exercise: Regulation and Integration of Multiple Systems: Oxford University Press, New York; 1996, p. 614-648.

3. Mader A, Liesen H, Heck H, Philippi H, Rost R, Schurch P, Hollman W. Zur Beurteilung der Sportartspezifischen aus Dauerleistungsfahigkeit im Labor. Sportarzt Sportmed 1976; 27: 80-88.

4. Fontana P, Boutellier U, Knopfli-Lenzin C. Time to exhaustion at maximal lactate steady state is similar for cycling and running in moderately trained subjects. Eur J Appl Physiol 2009; 107 (2): 187-192.

5. Bosquet $\mathbf{L}$, Leger $\mathbf{L}$, Legros $P$. Methods to determine aerobic endurance. Sports Med 2002; 32 (11): 675-700.

6. MacIntosh BR, Shahi MR. A peripheral governor regulates muscle contraction. Applied physiology, nutrition, and metabolism. Physiol Appl Nutrit Metab 2011; 36 (1): 1-11.

7. Coyle EF, Coggan AR, Hemmert MK, Ivy JL. Muscle glycogen utilization during prolonged strenuous exercise when fed carbohydrate. J Appl Physiol 1986; 61 (1): 165-172.

8. Febbraio MA, Dancey J. Skeletal muscle energy metabolism during prolonged, fatiguing exercise. J Appl Physiol 1999; 87 (6): 2341-7.

9. Beneke R, Hutler M, Leithauser RM. Maximal lactate-steady-state independent of performance. Med Sci Sport Exercise 2000; 32 (6): 1135-1139.

10. Baron B, Dekerle J, Depretz S, Lefevre T, Pelayo P. Self selected speed and maximal lactate steady state speed in swimming. J Sport Med Phys Fitness 2005; 45 (1): 1-6.

11. McArdle WD, Montoye HJ. Reliability of exhaustive swimming in the laboratory rat. J Appl Physiol 1966; 21 (4): 1431-1434.

12. Beck WR, de Araujo GG, Gobatto CA. Methods of exercise intensity and lactataemia determination of lactate minimum test in rats. Comp Exercise Physiol 2012; 8 (2): 113-116.

13. de Araujo GG, Papoti M, Manchado Fde B, de Mello MA, Gobatto CA. Protocols for hyperlactatemia induction in the lactate minimum test adapted to swimming rats. Comp Biochem Physiol Part A, Mol Integr Physiol 2007; 148 (4): 888-892.

14. Gobatto CA, de Mello MA, Sibuya CY, de Azevedo JR, dos Santos LA, Kokubun E. Maximal lactate steady state in rats submitted to swimming exercise. Comparative biochemistry and physiology Part A, Mol Integr Physiol 2001; 130 (1): 21-27.

15. Manchado FB, Gobatto CA, Contarteze RVL, Papoti M, Mello MAR. Maximal lactate steady state in running rats. J Exerc Physiol 2005; 8: e29-35.

16. Tanaka H, Yanase M, Kanosue K, Nakayama T. Circadian variation of thermoregulatory responses during exercise in rats. Amer J Physiol 1990; 258 (4 Pt 2): R836-841.

17. Sun JH, Yaga K, Reiter RJ, Garza M, Manchester LC, Tan DX, Poeggeler B. Reduction in pineal N-acetyltransferase activity and pineal and serum melatonin levels in rats after their exposure to red light at night. Neurosci Lett 1993; 149 (1): 56-58. 
18. Pittendrigh CS, Daan SA. A functional analysis of circadian pacemakers in nocturnal rodents. IV. Entrainment: pacemaker as clock. J Comp Physiol 1976; 106: 291-331.

19. Engel PC, Jones JB. Causes and elimination of erratic blanks in enzymatic metabolite assays involving the use of NAD + in alkaline hydrazine buffers: improved conditions for the assay of L-glutamate, L-lactate, and other metabolites. Analyt Biochem 1978; 88 (2): 475-484.

20. Dubois ML, Gilles KA, Hamilton JK, Rebbers PA, Smith F. Colorimetric method for determination of sugars and related substances. Anal Chem 1956; 28: 350-356.

21. Baron B, Noakes TD, Dekerle J, Moullan F, Robin S, Matran R, Pelayo P. Why does exercise terminate at the maximal lactate steady state intensity? Brit J Sport Med 2008; 42 (10): 828-833.

22. Oliveira CA, Paiva MF, Mota CA, Ribeiro C, Leme JA, Luciano E, Mello MA. Exercise at anaerobic threshold intensity and insulin secretion by isolated pancreatic islets of rats. Islets 2010; 2 (4): 240-246.

23. Luciano E, Mello MAR. Effects of chronic physical exercise on protein levels in diaphragm from diabetic rats. Motriz 1999; 5: 146-151.

24. Rossi L, Tirapegui J. Currents concepts about physical exercise, fatigue and nutrition. Braz J Phys Educ Sport 1999; 3: 67-82.

25. Kuipers H. Training and overtraining: an introduction. Med Sci Sport Exercise 1998; 30 (7): 1137-1139.

26. Wegener G, Krause U, Newsholme EA. Metabolic regulation-physiological and medical aspects. Experientia 1996; 52 (5): 391-395.
27. Curi R, Lagranha CJ, Jair Rodrigues G, Pithon-Curi TC, Lancha Jr AH, Pellegrinotti IL, Procópio J. The Krebs Cycle as Limiting Factor for Fatty Acids Utilization During Aerobic Exercise. Braz Arch Endocr Metab 2003; 47: 135-143.

28. Powers SK, Howley ET. Exercise physiology: theory and application to fitness and performance. Madison: Brown \& Benchmark Publishers 1994.

29. Green HJ. How important is endogenous muscle glycogen to fatigue in prolonged exercise? Canad J Physiol Pharmacol 1991; 69 (2): 290-297.

30. Ament W, Verkerke GJ. Exercise and fatigue. Sports Med 2009; 39 (5): 389-422.

31. Peter JB, Barnard RJ, Edgerton VR, Gillespie CA, Stempel KE. Metabolic profiles of three fiber types of skeletal muscle in guinea pigs and rabbits. Biochem 1972; 11 (14): 2627-2633.

32. Armstrong RB, Phelps RO. Muscle fiber type composition of the rat hindlimb. Amer J Anat 1984; 171 (3): 259-272.

33. Armstrong RB, Laughlin MH. Metabolic indicators of fibre recruitment in mammalian muscles during locomotion. J Exp Biol 1985; 115: 201-213.

34. Peterson DF, Armstrong RB, Laughlin MH. Sympathetic neural influences on muscle blood flow in rats during submaximal exercise. J Appl Physiol 1988; 65 (1): 434-440.

35. Armstrong RB, Laughlin MH. Blood flows within and among rat muscles as a function of time during high speed treadmill exercise. J Appl Physiol 1983; 344: 189-208. 